\title{
Purification and Characterization of Anti-complementary Polysaccharide from Leaves of Thymus vulgaris $\mathrm{L}$.
}

\author{
Hyug Chun, Woo Jin Jun, Dong Hoon Shin, Bum Shik Hong, ${ }^{*}$ Hong Yon Cho, and Han Chul Yang \\ Graduate School of Biotechnology, Korea University, \# 1, 5-Ka, Anam-Dong, Sungbuk-Ku, Seoul, Korea 136-701. \\ Received December 4, 2000; accepted March 10, 2001
}

For the purification of the anti-complementary polysaccharide from Thymus vulgaris L., the hot-water extract of thyme leaves was successively fractionated by ethanol precipitation and ultra-filtration. The retentate with 300-kDa membrane cartridge showed a potent anti-complementary activity. It was further purified by open column chromatographies on DEAE-Toyopearl 650C, Butyl-Toyopearl 650M and Sephadex G-100, obtaining TV3-IIA-I, the purified anti-complementary polysaccharide. The anti-complementary polysaccharide exhibited the anti-complementary activity via both classical and alternative pathways. Based upon the methylation analysis and the reaction with $\beta$-glucosyl Yariv reagent, the anti-complementary polysaccharide from thyme might contain an arabinogalactan moiety, at least in part.

Key words Thymus vulgaris L.; anti-complementary polysaccharide; arabinogalactan

The leaves of thyme (Thymus vulgaris L.) have been used as tea and spice in western recipes to improve the taste of dishes. The aromatic compounds and oily components of thyme contain several pharmacological effects such as the inhibition of oxidation and breast cancer. ${ }^{1)}$ The methanol extracts of thyme have showed the anti-cancer effects by the enhancement of production of leukocyte and thrombocyte in the blood stream. ${ }^{2)}$ According to Okuda et al., ${ }^{3)}$ there was a significant correlation between the activation of the complement system and anti-cancer effect by the enhancement of a host defense mechanism. Even though a large variety of pharmacological activities of thyme extracts have been reported, studies on the polysaccharides from thyme have not been investigated. Therefore, studies on compounds, which can modulate the activity of the complement system, may be useful for alternation of the chemicals on cancer. In the present study, we dealt with the purification and characterization of the anti-complementary polysaccharide from a hot-water extract of thyme.

\section{Experimental}

Materials Leaves of Thymus vulgaris L were purchased from Hyangwon spice Co. (Seoul, Korea) and authenticated by Dr. Sang-In Shim at Seed Bank for Wild Herbaceous Plant Species, Korea University. Sheep red blood cell sensitized by IgM hemolysin (EA cell) for a measurement of anti-complementary activity was purchased from Biotest Co. (Tokyo, Japan). $\beta$-DGlucosyl Yariv reagent was obtained from Biosupplies Australia (Parkville, Australia). All others chemicals were analytical reagent-grade.

Isolation and Purification of Anti-complementary Polysaccharides Thyme $(500 \mathrm{~g})$ was refluxed with 101 of methanol, and the residue was extracted with 201 of distilled water at $100^{\circ} \mathrm{C}$ for $2 \mathrm{~h}$. After centrifugation, the supernatant was precipitated with a stepwise increase in ethanol added (30, $75,95 \%)$. The $75 \%$ ethanol precipitate (TV-75, $15.5 \mathrm{~g}$ ) was further fractionated by ultrafiltration with $100-$ and $300-\mathrm{kDa}$ membranes (Masterflex prep/scale-tff ${ }^{\mathrm{TM}}$, Millipore Co., Bedford, MA, U.S.A.). The active fraction, TV-3 (molecular mass $>300 \mathrm{kDa}, 11.2 \mathrm{~g}$ ) was obtained.

To purify anti-complementary polysaccharide, sequential column chromatographies were carried out with TV-3. Each fraction with a high anticomplementary activity was repeatedly sub-fractionated. TV-3 was applied to a DEAE-Toyopearl 650C (Tosoh Co, Japan) column $\left(\mathrm{Cl}^{-}\right.$form, $2.5 \times 20 \mathrm{~cm}$ ). The sample was first eluted with 4-volume of distilled water (TV-3-I), and then with the successive stepwise gradient of $0.15,0.3,0.45$, 1.0 and $2.0 \mathrm{M} \mathrm{NaCl}$ (TV-3-II, -III, IV, V, -VI). Each fraction was collected, dialyzed and lyophilized. TV-3-II with the highest activity and yield $(4.2 \mathrm{~g})$ was eluted with a linear gradient from $2.0 \mathrm{M}\left(\mathrm{NH}_{4}\right)_{2} \mathrm{SO}_{4}$ to distilled water on a Butyl-Toyopearl 650M (Pharmacia Ltd., Uppsala, Sweden) column

* To whom correspondence should be addressed. e-mail: bumshik@tiger.ac.k
$(2.4 \times 10 \mathrm{~cm})$, resulting in two fractions (TV-3-IIA and -IIB). The active fraction, TV-3-IIA (Yield; $1.2 \mathrm{~g}$ ), was passed through a Sephadex G-100 (Pharmacia Ltd., Uppsala, Sweden) column $(2.5 \times 95 \mathrm{~cm})$ using $0.2 \mathrm{M} \mathrm{NaCl}$ as an eluent, obtaining the purified anti-complementary polysaccharide (TV-3IIA-I, Yield; $0.6 \mathrm{~g}$ ).

General Analysis The total carbohydrate and uronic acid were measured by the phenol-sulfuric acid ${ }^{4)}$ and $m$-hydroxybiphenyl $1^{5}$ methods, using D-glucose and D-galacturonic acid as the respective standards. To determine structural sugars, monosaccharide alditol acetate was prepared using the method described by Jones and Albersheim. ${ }^{6}$ The alditol acetates were analyzed by GC on a HP-5890 Series II gas chromatography (Hewlett-Packard, U.S.A.) equipped with a SP-2380 capillary column $(0.2 \mu \mathrm{m}, 0.25 \mathrm{~mm} \times 30$ $\mathrm{m}$, Supelco Co., U.S.A.). GC conditions were as follows; injector temperature $\left(250{ }^{\circ} \mathrm{C}\right)$, splitless, $\mathrm{He}$, column temperature $\left(60^{\circ} \mathrm{C}\right.$ for $1 \mathrm{~min}$, $60 \rightarrow 220^{\circ} \mathrm{C}$ at $30^{\circ} \mathrm{C} / \mathrm{min}$, held for $12 \mathrm{~min}, 220 \rightarrow 250{ }^{\circ} \mathrm{C}$ at $8{ }^{\circ} \mathrm{C} / \mathrm{min}$ and held for $15 \mathrm{~min})$, detector temperature $\left(250^{\circ} \mathrm{C}\right)$. The $\mathrm{mol} \%$ of monosaccharide was calculated from peak areas and the molecular weight of the corresponding alditol acetates. HPLC (Waters 2690 HPLC series) was performed on a column of Shodex OHpak KB-805 (GPC type, $8 \times 300 \mathrm{~mm}$, Toshohaas Co.) equipped with Waters $410 \mathrm{RI}$ detector.

Methylation Analysis The purified polysaccharide, TV-3-IIA-I, was methylated according to the Hakomori method, ${ }^{7)}$ and the methylated products were recovered using a Sep-pak $\mathrm{C}_{18}$ cartridge (Waters Associates, Milford, MA, U.S.A.) by the procedure of Waeghe et al. ${ }^{8}$ Carboxyl groups in methylated products were reduced with $\mathrm{Li}(\mathrm{Et})_{3} \mathrm{BD}_{3}$ in tetrahydrofuran (THF) (Super-Deuteride ${ }^{\mathbb{Q}}$, Aldrich Co., Milwaukee, WI, U.S.A.) and the partially methylated alditol acetates were prepared. ${ }^{6}$ The resulting partially methylated alditol acetates were analyzed by GC and GC-MS (Hewlett-Packard 5890 mass spectrometer). Partially methylated alditol acetates were identified by the fragment ions in MS and relative retention time in GC. The molar percentage was calculated from the peak area and response factor. ${ }^{9)}$

Anti-complementary Activity The inhibition of total complement hemolysis of control $\left(\mathrm{ITCH}_{50} \%\right)$ was measured with EA cell by the multi-tube system according to Kabat and Mayer. ${ }^{10)}$ For the determination of alternative complement hemolysis of control $\left(\mathrm{IACH}_{50} \%\right)$, the same procedure described above was performed, except for rabbit erythrocytes instead of EA cell. All data were expressed as mean \pm S.D. of three experiments, and the significance of differences between the means was evaluated by Student $t$-test.

\section{Results and Discussion}

The Purification of Anti-complementary Polysaccharide from Thyme The leaves of thyme were refluxed with methanol, and then the insoluble portion was extracted with hot water. The hot-water extract with a potent anti-complementary activity was further fractionated by ethanol precipitation. The highest anti-complementary activity was observed in TV-75, a 75\% ethanol precipitate, and it was subfractionated by ultrafiltration. After ultrafiltration, a high- 


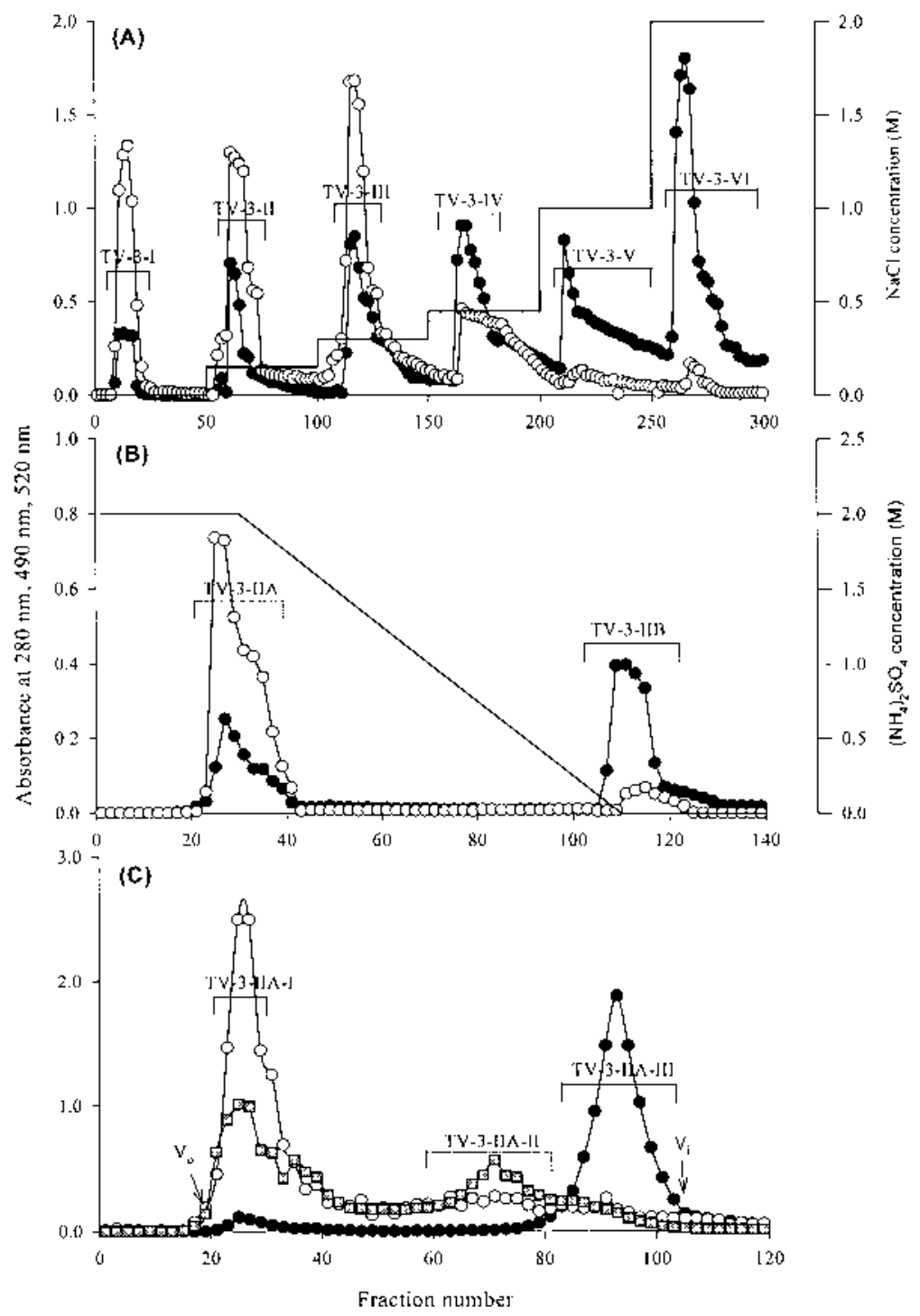

Fig. 1. Chromatograms of Each Purification Step of Crude Polysaccharide (TV-3) from Thymus vulgaris L.

(A) Ion-exchange chromatogram of TV-3 on DEAE-Toyopearl $650 \mathrm{C}$ column $\left(\mathrm{Cl}^{-}\right.$from, $\left.2.5 \times 20 \mathrm{~cm}\right)$ eluted with the successive stepwise gradient of NaCl solution with a fraction size of $8 \mathrm{ml}$ at a flow rate of $2 \mathrm{ml} / \mathrm{min}$. (B) Hydrophobic interaction chromatography of TV-3-II on Butyl-Toyopearl $650 \mathrm{M}$ column $(2.4 \times 10 \mathrm{~cm}$ ) eluted with a linear gradient from ammonium sulfate $(2.0 \mathrm{M})$ to distilled water (D.W.) with a fraction size of $5 \mathrm{ml}$ at a flow rate of $3 \mathrm{ml} / \mathrm{min}$. (C) Gel filtration chromatography of TV-3-IIA on Sephadex G-100 column $(2.5 \times 95 \mathrm{~cm})$ eluted with $200 \mathrm{~mm} \mathrm{NaCl}$ solution with a fraction size of $4 \mathrm{ml}$ at a flow rate of $0.2 \mathrm{ml} / \mathrm{min}$. $\bigcirc: A_{490 \mathrm{~nm}}, \mathbf{Q}: A_{280 \mathrm{~nm}}$, $\mathbb{F}_{\mathbf{F}}: A_{520 \mathrm{~nm}}, V_{\mathrm{o}}$ : void volume, $V_{\mathrm{i}}:$ inner volume.

molecular size polysaccharide (TV-3; $>300 \mathrm{kDa}$ ) showed the relatively high activity. For the purification of an anti-complementary polysaccharide, TV-3 was subjected to a DEAEToyopearl column, resulting in 6 subfractions (Fig. 1A). Of those, TV-3-II and TV-3-III exhibited the higher anti-complementary activities. We chose TV-3-II over TV-3-III for the further purification by the consideration of yield-wise $(4.2 \mathrm{~g}$ vs. $2.8 \mathrm{~g}$ ). Thus, TV-3-II was loaded on a Butyl-Toyopearl $650 \mathrm{M}$ column to exclude hydrophobic matters, obtaining TV3-IIA (Fig. 1B). An anti-complementary fraction (TV-3-IIA) was applied to a size exclusion column chromatography on Sephadex G-100. A relatively high-molecular size polysaccharide (TV-3-IIA-I) displayed the highest anti-complementary activity (Fig. 1C). HPLC profile indicated that TV-3IIA-I was highly purified (data not shown).

Mode of Anti-complementary Activity of TV-3-IIA-I The anti-complementary activity of TV-3-IIA-I was investigated via which pathway the purified polysaccharide acti- vated complement system. For the determination of an alternative pathway activity, $\mathrm{Mg}^{2+}$-EGTA (ethylene glycol-bis $(\beta$ aminoethylether) $N, N, N^{\prime}, N^{\prime}$-tetraacetic acid) $\mathrm{GVB}^{2-}$ and rabbit erythrocytes were used. TV-3-IIA-I expressed dose-dependent pattern in $\mathrm{ITCH}_{50}(\%)$. At the same concentration, higher value of $\mathrm{ITCH}_{50}(\%)$ was found with TV-3-IIA-I than Polysaccharide-K (PSK), which is a known activator of classical pathway. TV-3-IIA-I also showed alternative pathway activity, although the value of $\mathrm{IACH}_{50}(\%)$ was slightly lower than that of zymosan, an activator of alternative pathway found from yeast cell wall (Fig. 2). These results indicated that the purified polysaccharide activated the anti-complementary activity via both pathways.

Structural Characteristics of Anti-complementary Polysaccharide In general, anti-complementary polysaccharides derived from plant represent the highly complexed structure such as the polysaccharides from Angelica acutiloba KITAGAWA, ${ }^{11)}$ which are composed of pectin, arabino- 


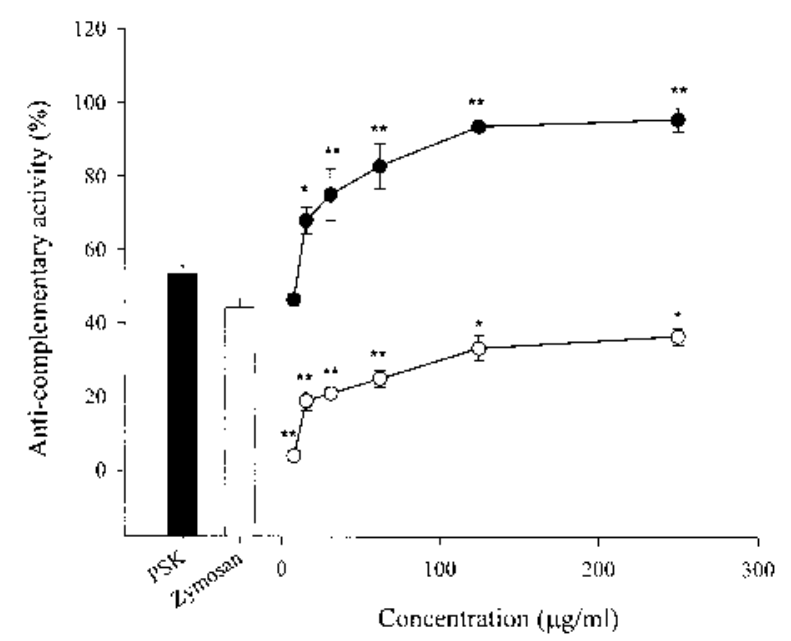

Fig. 2. Anti-complementary Activity of Purified Polysaccharide from Thymus vulgaris $\mathrm{L}$.

PSK (polysaccharide-K) and zymosan were used as positive references at $1000 \mu \mathrm{g} / \mathrm{ml} . * p<0.05$ and $* * p<0.01$ : significantly different from positive references. : $\mathrm{ITCH}_{50}(\%), \mathrm{O}: \mathrm{IACH}_{50}(\%)$.

galactan and galactan.

When TV-3-IIA-I was analyzed by GC and GC-MS, it contained $41.3 \mathrm{~mol} \%$ galactose, $15.6 \mathrm{~mol} \%$ arabinose, $13.6 \mathrm{~mol} \%$ mannose, $12.6 \mathrm{~mol} \%$ glucose, $9.9 \mathrm{~mol} \%$ rhamnose, $4.9 \mathrm{~mol} \%$ galacturonic acid and minors of xylose, fucose, glucuronic acid (data not shown). The molar ratio of arabinose and galactose was $0.4: 1.0$ of the purified polysaccharide. The methylation analysis indicated that TV-3-IIA-I was composed of various glycosidic linkages such as terminal galactose, 4-linked galactose, 3,6-branched galactose, 2,3,4-branched mannose, terminal arabinose, 4- or 5-linked arabinose, 4,6-branched glucose, 4-linked galacturonic acid and 2-linked rhamnose (Table 1). Arabinogalactans are classified into three groups (type I, II, III). By the specificity of $\beta$-glucosyl Yariv reagent to arabino-3,6-glactan, an attempt to react the reagent with TV-3-IIA-I was made, resulting in the strong reactivity (data not shown), which suggested the existence of an arabino-3,6-galactan portion $^{12)}$ in TV-3-IIA-I. It was supported by the presence of 4-linked galactose, 3,6branced galactose, and 4 or 5-linked arabinose and terminal arabinose. A deeper understanding of the relationship between structure and activity of the anti-complementary polysaccharide is needed. In current, the researches on anti-cancer effects of the anti-complementary polysaccharides from
Table 1. Methylation Analysis of the Purified Anti-complementary Polysaccharide from Thymus vulgaris $\mathrm{L}$.

\begin{tabular}{llr}
\hline \hline Glycosyl residue & Deduced glycosidic linkage & $\mathrm{mol} \%$ \\
\hline Rhamnose & 2 & 4.2 \\
& 4 & 0.5 \\
Fucose & 2,3 & 0.5 \\
Arabinose & 2 & 3.1 \\
& Terminal (furanosyl) & 4.0 \\
& 4 or 5 & 5.9 \\
& 2,3 & 0.5 \\
Xylose & 2,5 & 0.9 \\
Galactose & 3,5 & 1.4 \\
& 4 or 5 & 5.4 \\
& Terminal & 8.4 \\
& 3 & 1.7 \\
Mannose & 4 & 24.7 \\
Glucose & 6 & 3.0 \\
Galacturonic acid & 3,6 & 4.6 \\
& $2,3,4$ & 12.3 \\
Glucuronic acid & 4,6 & 7.3 \\
& $2,4,6$ & 1.6 \\
& 4 & 5.9 \\
& $2,3,4$ & 3.7 \\
\end{tabular}

thyme are in progress.

Acknowledgements This work was supported by grants from the agricultural R \& D promotion center of Korea.

\section{References}

1) Zava D. T., Dollbaum C. M., Blen M., Proc. Soc. Exp. Biol. Med., 217, 369-378 (1998).

2) Von Ardenne M., Reitnouer P. G., Pharmazie, 36, 703-705 (1981).

3) Okuda T., Yoshioka Y., Ikekawa T., Chihara G., Nishioka K., Nature New Biol., 238, 59-60 (1972).

4) Dubios M., Gilles K. A., Hamilton J. K., Rebers P. A., Smith F., Anal. Chem., 28, 350-356 (1956).

5) Blumenkranz N., Ashoe-hansen G., Anal. Biochem., 54, 484-489 (1973).

6) Jones T. M., Albersheim P., Plant Physiol., 49, 926-936 (1972).

7) Hakomori S., J. Biochem. (Tokyo), 55, 205-208 (1964).

8) Waeghe T. J., Darvill A. G., McNeil H., Albersheim P., Carbohydr. Res., 123, 281-304 (1983).

9) York W. S., Darvill A. G., McNeil M., Stevenson T. T., Albersheim P., Methods Enzymol., 118, 3- 40 (1986).

10) Kabat E. A., Mayer M. M. (eds.), "Experimental Immunochemistry," Charles and Thomas Publisher, Illinois, 1964, pp. 133-240.

11) Yamada H., Carbohydr. Polymers, 25, 269-276 (1994).

12) Clarke A. E., Anderson R. L., Stone B. A., Phytochemistry, 18, 521540 (1979). 